\title{
LOPES : Selective control of gait functions during the gait rehabilitation of CVA patients.
}

\author{
R. Ekkelenkamp J. Veneman H. van der Kooij \\ Institute for Biomedical Technology \\ Biomechanical Engineering, Department of Engineering Technology, University of Twente \\ PO box 2177500AE Enschede \\ The Netherlands
}

\begin{abstract}
LOPES aims for an active role of the patient by selective and partial support of gait functions during robotic treadmill training sessions. Virtual Model Control (VMC) was applied to the robot as an intuitive method for translating current treadmill gait rehabilitation therapy programs into robotic rehabilitation therapy. Virtual models are proposed for the selective control of gait functions during treadmill training. From this collection of models several, representing the extremes of the entire set of virtual models, were implemented. The results show that $\mathrm{VMC}$ is a promising method for the control of a gait rehabilitation robot.
\end{abstract}

\section{INTRODUCTION}

The goal of the LOPES project (LOwer-extremity Powered ExoSkeleton) is to design and implement a gait rehabilitation robot for treadmill training. The target group consists of people who have suffered a stroke and have impaired motor control.

The main goals of LOPES are:

- Reduction of the physical load on the therapist patient;

- More efficient gait training for stroke patients;

- Selective support of gait functions;

- Therapist stays in charge of high-level decisions.

The mechanical construction should offer assistance in leg movements in the forward direction and in keeping lateral balance. Within the LOPES project, it has been decided to realize this by connecting the limbs of the patient to an 'exoskeleton' so that robot and patient move in parallel (Fig. 1).Most gait rehabilitation robots that are currently being developed $[1,2]$ focus on the support of the entire gait cycle.

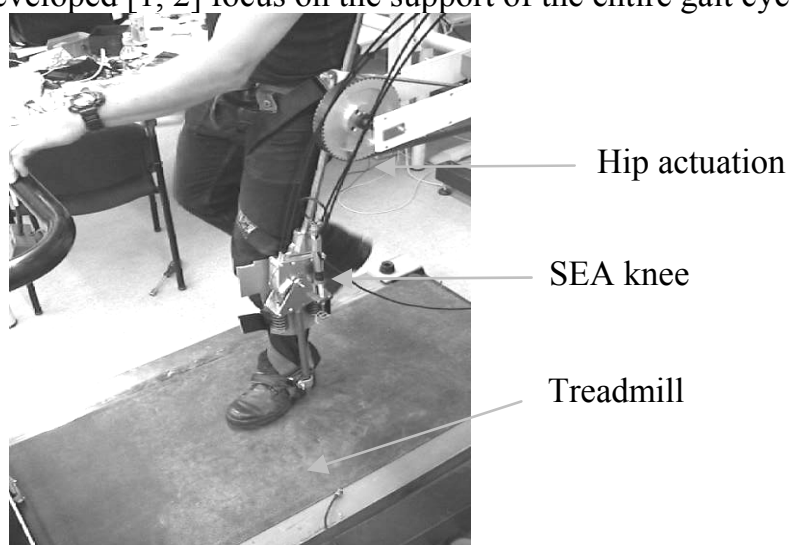

Figure 1: Current LOPES setup. This setup has 2DOF 1 in the hip and one in the knee of a single leg.
These robots use joint trajectories of the entire gait cycle and offer a uniform (more or less) stiff control along this trajectory. This means that the patient receives support in gait phases where support is necessary but also in phases where support isn't necessary. Studies have been done on the lokomat [3] that propose adaptive control methods which minimize the interaction forces with the patient with respect to an adaptable reference pattern, but these still control the entire gait cylce. Studies have also shown that walking with the current lokomat frame requires significantly less energy than normal walking [4]. This means that patients are not walking as actively as possible but are able to walk a greater distance. LOPES aims to support and not take over those tasks that the patient is unable to perform without help using an impedance control scheme. This will lead to a more active participation from the patient's side. The tradeoff for more active walking will likely be a smaller overall distance during therapy sessions. The implication of selective function support is that the robot will have two extreme modes in which it should be able to function, these are:

Patient in charge: The goal of the robot is to minimize the interaction forces between the patient and the robot in order for the patient to walk freely without feeling the robot. This mode will be active mostly for the non-paretic side of the patient and during those phases of the walking cycle that the robot does not need to assist.

Robot in charge: The goal of this mode is to take control of the patient. The robot will take over the functions which the patient is unable to perform.

The robot will most likely operate somewhere between these extremes offering some support at those phases when it is needed to guide the patient towards desirable behavior.

The control of LOPES will consist of three levels (Figure 2). The top layer, the observer, is the decision maker. This part will determine the current gait phase in which the patient is in and what support is needed in order for the patient to walk safely.

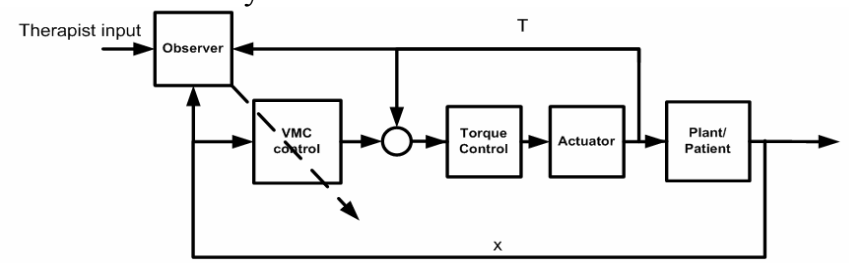

Figure 2: Control scheme of LOPES. The Observer controls which VMC is active at a certain time. The VMC controls the interaction of the patient and the torques are controlled separately for each joint. 
The second level, the main focus of this paper, describes the interaction forces between the patient and the robot. We have chosen for Virtual Model Control (VMC), which has successfully been applied in walking robots [5]. Some benefits of Virtual Model Control are that it is compact, requires relatively small amounts of computation, and can be implemented in a distributed manner. VMC control is also well suited for an intuitive translation of physical interactions (of the therapist) to a relatively simple control scheme. In addition, it can be used to implement robust and compliant controls intuitively providing safe and gentle control. The basis of this control method is to define physical interactions with the subject that would result in the required rehabilitation e.g. maintaining balance for the patient and preventing stumbles. These interactions are then translated into a set of Virtual physical Models (VMs) such as springs and dampers (Fig. 3) that can be switched on and off at appropriate times in the gait cycle by the observer (Fig. 2). The desired stiffness and damping of the virtual models are related to the amount of support, and are not necessary linear but can depend on time or position. The virtual forces that would be exerted by interaction between the virtual models and the subject are translated to joint torque commands for the joint actuators. This transformation can be written as:

$$
\tau=J^{T} \cdot F
$$

With $\tau$ being the joint torque vector, $\mathrm{J}$ is the Jacobian matrix of the exoskeleton, and $\mathrm{F}$ the virtual Forces vector. The torque for each joint is controlled separately. The bandwidths of the VMC controllers are at least a factor 3 lower for stability requirements.

The goal of this article is to show that VMC can be used to selectively support gait during treadmill training. We propose several VMs that could be used to control a patients gait based on observations made during rehabilitation sessions in rehabilitation centers in the Netherlands. Several of these proposed VMs have been implemented in a LOPES test frame to evaluate experimentally whether the desired minimal and maximal impedance and the bandwidth of VMC can be achieved. The remaining VMs have been implemented only on a theoretical model of a completely passive human being in the frame (results will not be shown here). As a first proof viability of VMC in this application, we demonstrate that the foot clearance of a person walking in the test frame can be effectively modulated with VMC.

\section{REHABILITATION SESSIONS - INTERVENTIONS AND PROPOSED VIRTUAL MODELS}

This section will describe the different therapeutic goals and a translation into virtual models (VMs). The therapeutic goals we consider are:

1. Unhindered walking;

2. Postural stability of the patient;

3. Sufficient foot clearance;

4. Support weight Bearing;

5. Increase speed of walking.
Each of these interactions between the patient and the therapist is described and translated to a VM (Fig. 3). The VMs described here can guide the patient through the gait cycle. The therapist will be able to determine the set points and coefficients for the different VMs and thus determining the amount of support which is offered to the patient.

Goal 1: Unhindered walking. When the patient walks within the desired boundaries, (s)he should ideally not feel the robot.

LOPES: The robot needs to actively compensate for the weight and friction of the exoskeleton and reflected mass of the motors. The weight of the exoskeleton can be compensated by implementing non linear torsion springs (VMs not shown).

Goal 2: The balance of the patients has to be supported.

Therapist action: Guiding the foot of the paretic side of the patient during the swing phase to make sure the abduction of the hip is adequate to support the patient. For more seriously afflicted patients a second therapist holds on to the pelvis of the patient and guides the centre of mass along a desired trajectory.

LOPES: Lateral balance can be supported by a combination of two spring-dampers connected to the COM (VM1). Balance can also be supported more dynamically through adequate foot placement in the frontal and sagittal planes. Triggered and properly placed spring dampers can control the step length and width (e.g. VM2). Trunk balance can be supported by torsion spring damper combination attached to the trunk and the fixed world (VM not shown).

Goal 3: Sufficient foot clearance during swing phase.

Therapist action: The therapist bends the knee during the swing phase of the paretic leg by guiding both the foot and the ankle and a slight pressure at the back of the knee.

LOPES : A virtual granny walker attached at the ankle (VM3) will push the foot upwards when the foot comes to close to the ground in early and mid swing.



Figure 3: Example of Virtual Models (VMs) to support gait. VM 1 supports the balance of the patient. VM2 assist the patient in the placement of the foot in the sagittal and frontal plane, which is important for dynamic balance and the speed of walking. VM3 enforces sufficient foot clearance using a virtual granny walker connected at the ankle. VM4 helps to stabilize the knee. VM5 is a virtual granny walker (partial) supporting the patient's weight. VM6 increases the patient's push off. (*is implemented) 
Goal 4:The patients weight needs to be supported

Therapist action: In order to support the weight of the patient the therapist uses a harness with a suspension system. This can be a spring or counter balance system. Alternatively, the therapist can use his arm to stabilize the patient's knee.

LOPES: Whole body weight can be supported by implementing a virtual granny walker connected to the COM (VM 5). Implementing a virtual torsion spring on the knee just before loading stabilizes the knee (VM4).

Goal 5: Speed of walking should increase.

Therapist action: It is not viable for the therapist to offer push off support because of the high forces needed during this gait phase.

LOPES: The push off can be realized by a constant force pulling at the COM in the double support phase (VM6). Increasing or decreasing the step length (VM 2) can also slow down or speed up the patient's gait.

\section{METHODS}

The current LOPES frame is actuated only in the sagittal plane. In order to test actuation and VMC we have implemented a single leg. A Maxon EC45 motor connected to the hip axis through a timing belt controls the hip function. This motor is current controlled for the force control and there is no force feedback. The knee consists of a series elastic actuator. The specifications of this actuator are described in a parallel article [6] . The forces at the ankle of the exoskeleton, are measured using an ATI mini 456 DOF force/moment sensor. The ankle is not controlled due to several reasons: There is often very little improvement on the ankle function, secondly construction wise it is difficult to implement. Finally, during current treadmill training the ankle is not trained. The ankle when necessary will be locked in a neutral position.

In order to test whether the VMC is suitable to support gait and to walk unhindered in LOPES:

1. The minimal Cartesian impedance measured at the ankle joint of LOPES was determined. The weight of the exoskeleton was compensated as described in the previous section. The force sensor connected to the ankle was moved by hand along lines parallel to the horizontal and vertical axis. Forces were measured using the 6 DOF sensor between the user and the exo-skeleton.

2. The maximal stiffness at the ankle joint of LOPES was determined, which is a measure of how well LOPES will be able to support gait functions (e.g. weight bearing (VM5) or lateral postural stability (VM1). VM 5 was implemented and the force sensor was connected to the fixed world while pressing down the exoskeleton. The motion was so slow that it will be considered static;

3. The step response time was determined. The force build up should be fast enough to support the patient during quick events such as knee stabilization at impact (VM 4). The ability to produce a torque of $19.7 \mathrm{Nm}$ within 0.2 seconds would be suffucient [7] to support these events. The step response of the SEA was measured by applying a setpoint to the reference torque.

4. The foot clearance of a person walking in the test frame is modulated with VM3 as a first proof of principle

Note that 1 corresponds to the patient in charge mode and that 2-4 are versions of the robot (partial) in charge mode.

\section{RESUlts}

For the patient in charge mode the minimal Cartesian impedance of the ankle joint in the horizontal direction has been measured (Fig 4).

Up to the main frequency of CVA gait $(0.5 \mathrm{~Hz})$ the gain of the measured impedance remains constant. Above this frequency the impedance increases with a gradient corresponding with a mass roughly equal to the mass of the exo-skeleton $(3 \mathrm{~kg})$. In the time domain it can be seen that for lower frequencies forces remain below $10 \mathrm{~N}$ for frequencies below $1 \mathrm{~Hz}$. The maximum interaction force measured during the trials was $50 \mathrm{~N}$ for a $4 \mathrm{~Hz}$ movement. (results not shown). Several people have walked in LOPES in the patient in charge mode and reported that the system was not an encumbrance in the actuated DOF. However the fact that the robot does not allow abduction of the hip was considered to be an encumbrance. The impedance in the vertical impedance was measured and was found to be identical the horizontal direction (result not shown).



figure 4: The minimal impedance measured at the ankle was measured while the ankle was moved by hand along the horizontal axis. As can be seen the impedance stays constant up to $0.5 \mathrm{~Hz}$ (around $75 \mathrm{~N} / \mathrm{m}$ ). Above this frequency the impedance rises with mass characteristics (dashed line shows the equivalent mass of $3 \mathrm{~kg}$ ).
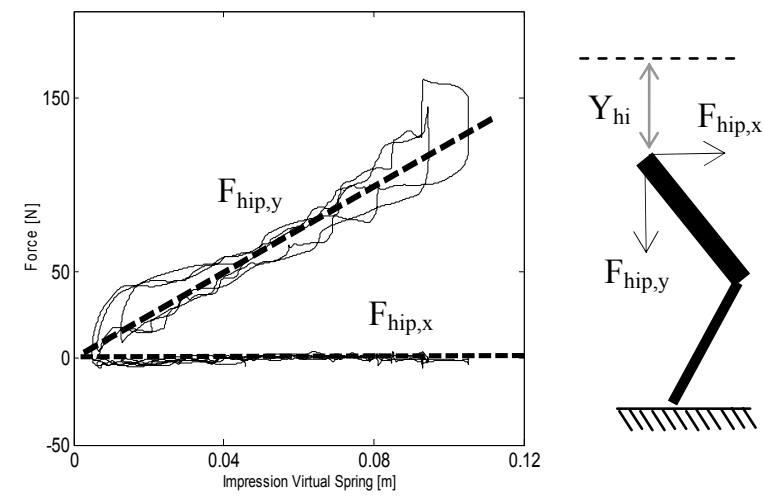

Figure 5: An example of selective stiffness $\left(\mathrm{K}_{\mathrm{y}}=1.25 \mathrm{kN}, \mathrm{Kx}=0\right)$ measured at the ankle using the 6DOF force sensor. The ankle was moved approx 10 $\mathrm{cm}$ in the vertical direction. There was an approximately $1 \mathrm{~cm}$ movement in the $\mathrm{x}$ direction. The dashed lines show the desired stiffness components. 
Figure 5 shows a representative stiffness curve for a vertical stiffness as described by VM5. The maximum stiffness that could be achieved for this VM was $10 \mathrm{kN} / \mathrm{m}$. VM 3 could effectively modulate the foot clearance (Fig.7). The subject walking in LOPES found that is was possible to walk normally in the unaffected phases. Beyond the discrete switching of the VMC when the ankle came below the given threshold the patient also found no problems with VM3.The response to a step input in the reference angle shows that the rise time is $0.05 \mathrm{sec}$ and the settling time is $0.1 \mathrm{sec}$ (Fig. 6).

\section{DisCUSSION}

The forces needed to move the exoskeleton in "patient in charge mode" are small (Fig. 4) for the main walking frequencies of CVA patients (up to $0.7 \mathrm{~Hz}$ ). Below $0.7 \mathrm{~Hz}$ the system behavior is dominated by the stiction and other unmodeled system attributes. Above these frequencies the behavior of the system is dominated by the mass of the exoskeleton. VMC could largely compensate the weight of the exoskeleton. Subject walking in the test frame did not perceive the remaining mass as a problem. The maximum obtained linear stiffness was $10 \mathrm{kN} / \mathrm{m}$. This means that a person weighing $100 \mathrm{~kg}$ and standing on one leg would drop $10 \mathrm{~cm}$ which is better than during current therapy.

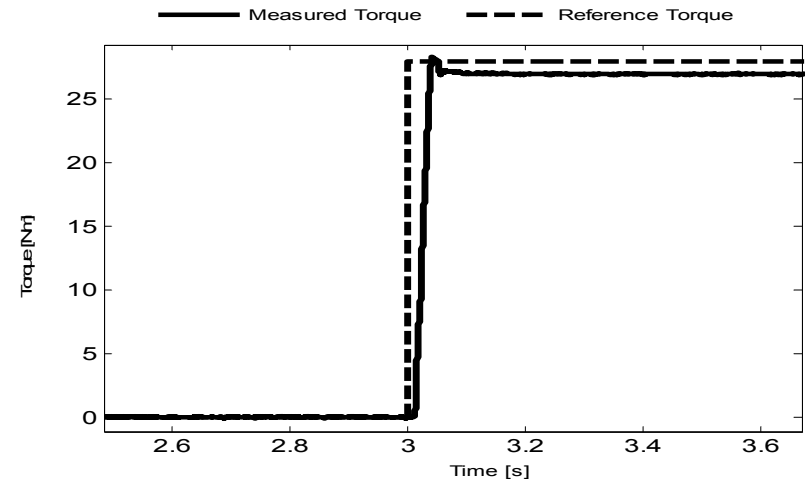

Figure 6: Force build up of the SEA in the knee.The steady state error is caused by the gravity working on the lower leg.

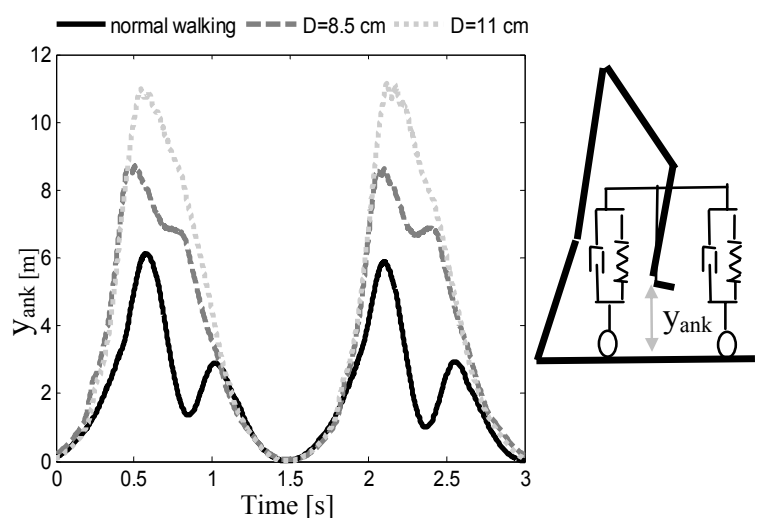

Figure 7: Ankle height with respect to the ankle position during stance during walking test. VM 3 was first switched off and the test-person was asked to walk normally. Next VMC3 was switched on with two different set points for the minimum foot clearance during the forwardmovement of the hip $\mathrm{D}=8.5 \mathrm{~cm}$ and $\mathrm{D}=11 \mathrm{~cm}$. The stance phase was not affected.
One problem is the accuracy in the low force ranges caused by the imperfect open-loop torque control of the hip joint.

In the subsequent version of the LOPES frame the hip will be replaced with a series elastic actuator. The small component in the horizontal can also be attributed the hip control. The system was able to support a subject without discomfort or large jerks.

In the future more VMs will be implemented to evaluate how well the therapeutic goals can be met. Adjusting the timings and physical properties of the VMs have to give insight in the most effective and comfortable properties of VMC applied to robotic aided gait rehabilitation. The current interface allows tuning of each parameter of each VM. This would make the system very complex and thus for a future version we will look into an intuitive interface with the therapist.

\section{CONCLUSION}

We have proposed a set of virtual models which could be used for the control of a gait robot. We have demonstrated that the minimum impedance of the test frame is low for the main gait frequencies. This was sufficient for the test persons to feel like they could walk with a normal gait. The force build up meets the bandwidth requirements of a gait rehabilitation device. We have shown that it is possible to selectively influence foot lift during the swing phase. This shows that VMC can be considered a promising option for the control of rehabilitation robots.

\section{ACKNOWLEDGEMENTS}

This research is supported by the NWO (Vernieuwingsimpuls 2001, granted to dr. H. van der Kooij) and by the institute for Biomedical Technology.

\section{REFERENCES}

1. Colombo, G., et al., Treadmill training of paraplegic patients using a robotic orthosis. Journal of Rehabilitation Research and Development, 2000. 37(6): p. 693-700.

2. Schmidt, H., et al., Development of a robotic walking simulator for gait rehabilitation. Biomedizinische Technik, 2003. 48(10): p. 281-286.

3. Jezernik, S., G. Colombo, and M. Morari, Automatic gait-pattern adaptation algorithms for rehabilitation with a 4-DOF robotic orthosis. Robotics and Automation, IEEE Transactions on, 2004. 20(3): p. 574.

4. C. Krewer, F.M., B. Husemann, S. Heller, J. Quintern, E. Koenig. Energy expenditure of hemiparetic patients and healthy subjects: walking in a lokomat vs. on a treadmill. in EvidenceBased Medicine in Neurorehabilitation. 2004. Zurich.

5. Pratt, J., et al., Virtual model control: An intuitive approach for bipedal locomotion. International Journal of Robotics Research, 2001. 20(2): p. 129-143.

6. J. Veneman, R.E., R. Kruidhof, F.C.T. van der Helm, H. van der Kooij, Design of a Series Elastic- and Bowdencable-based actuation system for use as torque-actuator in exoskeleton-type training robots. proceedings of the Icorr 2005, 2005.

7. Lelas, J.L., et al., Predicting peak kinematic and kinetic parameters from gait speed. Gait \& Posture, 2003. 17(2): p. 106. 